\title{
GlueX at Jefferson Lab: a search for exotic states of matter in photon-proton collisions
}

\author{
Matthew Shepherd* \\ Indiana University, Bloomington \\ E-mail: mashephedindiana. edu
}

\begin{abstract}
The GlueX Experiment, which is currently under construction as a component of the $12 \mathrm{GeV}$ upgrade to Jefferson Lab, will utilize photoproduction on a proton target to search for hybrid mesons in the light quark sector. Recent first-principles calculations of the hadron spectrum in Quantum Chromodynamics suggest the presence of bound states in the meson spectrum that cannot arise from a quark and an anti-quark. Such states appear to have valance gluonic content or gluonic degrees of freedom and are called hybrid mesons. An interesting subset of these, the "exotic hybrid mesons," have total angular momentum, parity, and charge conjugation quantum numbers that cannot be formed with a pair of spin-1/2 fermions. By performing an amplitude analysis of photon-proton reactions, the GlueX experiment will attempt to experimentally establish the spectrum of hybrid mesons. In this article, the present theoretical and experimental landscape is reviewed, the design of the GlueX detector presented, and the GlueX startup plans are briefly discussed.
\end{abstract}

52 International Winter Meeting on Nuclear Physics - Bormio 2014,

27-31 January 2014

Bormio, Italy

\footnotetext{
* Speaker.
} 


\section{Introduction}

The underlying color symmetry of Quantum Chromodynamics (QCD) gives rise to unique properties of the strong interaction. Preservation of local gauge invariance under rotations in color space requires that force-mediating gluons interact with each other. These gluon-gluon interactions are at the heart of many interesting phenomena in QCD. In particular the emergence of gluon loops from the vacuum lead to an anti-screening of the strong force at larger distances and low energies, the result of which seems to be that there are no free quarks: all known, isolated QCD states are color singlets. There are multiple experimental routes to study QCD. One can, for example, study hadron structure which is probing matter at low energy and large distance: the strongly coupled regime of QCD. The GlueX experiment seeks to study QCD through spectroscopy: studying the spectrum of QCD bound states that arise by combining quarks and gluons in various combinations. By observing patterns in this spectrum one can learn about the fundamental constituents of matter and the forces that bind them, i.e., quark and gluon interactions in QCD.

The gluon-gluon interaction could give rise to bound states of gluons, called "glueballs," or quark-gluon "hybrid" mesons. Both types of states have been the subject of experimental searches for several decades (see Ref. [1] for a review). The search for glueballs is complicated by the fact that the lightest glueball candidates are expected to have the same quantum numbers: total angular momentum $J$, parity $P$, and charge conjugation $C$ as ordinary meson states. Therefore, they may mix with meson states, and the glueball content can only be deduced by examining the dynamical properties of the state such as production and decay modes. A subset of the hybrid mesons, however, have $J^{P C}$ combinations that cannot be created by a fermion-antifermion pair. This provides a unique experimental signature: any state with exotic $J^{P C}$ cannot be a conventional quark anti-quark meson. One of the primary objectives of the GlueX experiment at Jefferson Lab is to search for and study such exotic hybrid mesons. These states are unique manifestations of the gluon-gloun coupling in QCD.

\section{Theoretical Context}

A crucial element of conducting a quantitative test of our understanding of the strong interaction in the strongly coupled regime is the ability to calculate experimental observables directly from QCD. In hadron spectroscopy, models of QCD have been very successful. In fact, the simple quark model, where mesons are composed of a quark and antiquark and baryons are composed of three quarks, does an outstanding job of describing masses and dynamical properties of of almost every observed hadron. It naturally leads to a question: why does QCD, which, in principle, permits a state of any color singlet combination of quarks and gluons, appear to have a spectrum that can so simply be described by quark-antiquark and three-quark states? QCD models such as the "bag model" [2, 3, 4] and the "flux-tube model" [5] and others have predicted a richer spectrum of hadrons with gluonic excitations for many years. However, the lack of experimental evidence for such states may lead one to question the accuracy of QCD models.

Over the last decade, a set of computational techniques known broadly as "Lattice QCD" has emerged as one viable method of performing first principles calculations of the hadron spectrum in QCD. A compilation of lattice QCD calculations by the Hadron Spectrum Collaboration [6] is 


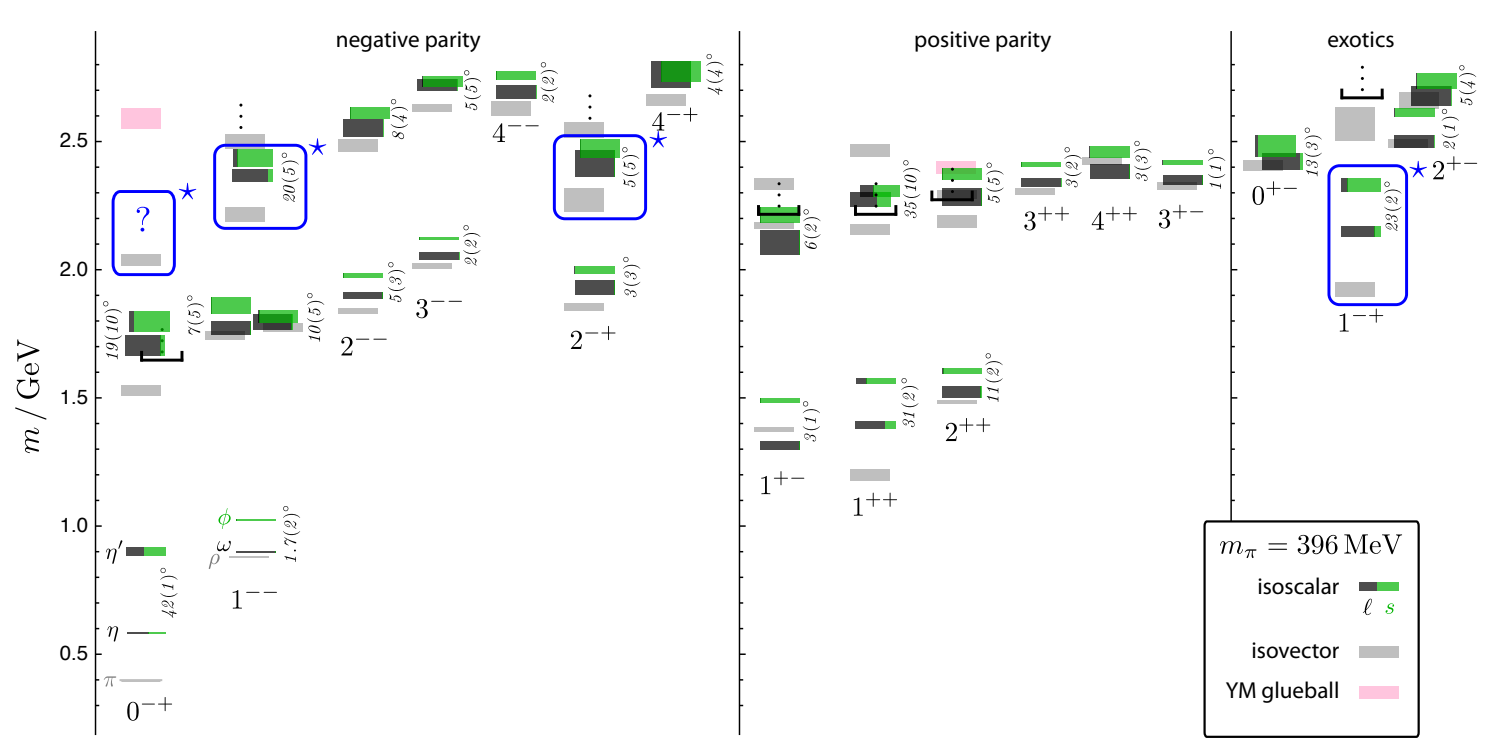

Figure 1: A compilation of lattice QCD calculations of the spectrum of light meson states [6]. The blue boxes indicate those states consistent with the lightest hybrid supermultiplet.

shown in Fig. 1. The lightest states have been labelled with and one can see that the pattern of low-lying states matches that observed by experiment and predicted by the quark model. Note that the $s \bar{s}$ component of isoscalars is also computed, and agrees with experimental observation, e.g., the $\eta$ and $\eta^{\prime}$ are nearly maximally mixed while the $\phi$ and $\omega$ are almost pure states. This calculation predicts a rich spectrum of hybrid mesons. One sees a supermultiplet of $0^{-+}, 1^{-+}, 2^{-+}$, and $1^{--}$ states (boxed in blue), all of which have significant gluonic content. The spectrum of hybrids in lattice QCD is largely consistent with what expects by coupling a $P$-wave gluon with $J_{g}^{P_{g} C_{g}}=1^{+-}$ to a color-octet $q \bar{q}$ pair. For the lightest hybrids that $q \bar{q}$ pair is in an $S$-wave with the spin-triplet producing the $0^{-+}, 1^{-+}$, and $2^{-+}$and the spin-singlet giving rise to the $1^{--}$. For each $J^{P C}$ one expects an isovector triplet and two isoscalars. Of the lightest hybrid states the $1^{-+}$have exotic $J^{P C}$; the three predicted states would be named the $\pi_{1}, \eta_{1}$, and $\eta_{1}^{\prime}$. The spectrum of heavier hybrids and exotics is consistent with coupling $P$-wave gluon to a $P$-wave $q \bar{q}$ pair.

These exotic configurations, the $1^{-+}$and the slightly heavier $0^{+-}$and $2^{+-}$states, the latter of which should have a pair of exotics for each of the isovector and two isoscalar quark configurations, are the prime focus of the GlueX experiment. Detection of exotic hybrids at GlueX requires not only that they exist, which lattice QCD calculations seems to strongly predict, but also that they can be produced in photoproduction, have widths comparable to conventional mesons, and decay to final states that are reconstructable by the detector. Calculation of couplings necessary to predict partial widths and production rates is in active of development in lattice QCD; no results for light quark hybrids exists as of yet. Nevertheless, the unique feature of photoproduction is that the photon beam acts a virtual vector meson, the quark model description of which puts the quarks in a spin-triplet configuration instead of the spin-singlet configuration one would obtain with a pion 
beam. This has led to predictions of large exotic hybrid photoproduction cross sections in some models [7]. Meson photoproduction at this energy regime remains largely unexplored; GlueX is positioned to conduct a definitive search for hybrids in photoproduction that is complementary to high-statistics hadron beam efforts carried out by E852 and COMPASS.

\section{Experimental Context}

While the existence of hybrid mesons has been suggested for many decades and numerous experimental searches have been conducted, the experimental evidence for such states is intriguing but somewhat inconclusive. One certain statement can be made: the rich pattern of states predicted by lattice QCD has not yet been observed by experiment. To date, most experimental evidence concerns searches for the lightest isovector hybrid meson, the $\pi_{1}$. A comprehensive review of the experimental status of searches for hybrids can be found in Ref. [8].

One of the most intriguing pieces of evidence comes from an observation by the E852 Collaboration of $\pi_{1}(1600) \rightarrow \eta^{\prime} \pi^{-}$in pion-proton collisions [9]. In this case the exotic $P$-wave amplitude is of equal or larger size as the $D$-wave amplitude that contains the $a_{2} \rightarrow \eta^{\prime} \pi$ decay. The CLEO collaboration also observed evidence for intensity in the exotic $1^{-+} \eta^{\prime} \pi$ amplitude in the decay $\chi_{c 1} \rightarrow \eta^{\prime} \pi^{+} \pi^{-}$[10], which could be described a resonant structure with properties consistent with that observed by E852.

The $\rho \pi$ decay mode has also been used to search for the $\pi_{1}$. Recent evidence of the $\pi_{1}(1600)$ comes from a high-statistics study of pion-lead collisions by the COMPASS collaboration, in which a signal for exotic $\pi_{1}(1600) \rightarrow \rho \pi$ is reported [11]. The CLAS Collaboration has conducted searches for the $\pi_{1}(1600) \rightarrow \rho \pi$ in photoproduction at $6 \mathrm{GeV}$ energies and finds no evidence for the state [12]. From this one can only limit the product of the photoproduction cross section of $\pi_{1}(1600)$ times the branching fraction of $\pi_{1}(1600) \rightarrow \rho \pi$; drawing a firm conclusion about the properties of the $\pi_{1}(1600)$ or photoproduction of hybrids in general is challenging. A common feature of analyses of the $3 \pi$ channel is that one must separate a signal for the exotic state from the conventional $\pi_{2}(1670)$ which has approximately the same mass and width as the exotic candidate and decays dominantly to $3 \pi$ through the $f_{2} \pi$ and $\rho \pi$ channels. The $\eta^{\prime} \pi$ channel has no such background.

The interest in unconventional mesons has peaked dramatically in the last decade with many recent discoveries in the charmonium system. (A thorough description of the current status of charmonium spectroscopy goes beyond the scope of this work; for more details see Refs. [13, 14] and the references therein.) As in the light quark system, lattice QCD calculations predict a spectrum of charmonium hybrid mesons [15]. The fact that one expects a vector $\left(1^{--}\right)$charmonium hybrid with a mass approximately equal to that of the mysterious $Y(4260)$ has fueled speculation about the nature of the later and motivated additional experimental studies. Seeking to understand the $Y(4260)$ better, the BESIII collaboration collected data at $e^{+} e^{-}$center-of-mass energies around $4260 \mathrm{MeV}$. Subsequent analysis led to the discovery of multiple heavy, charged structures [16, 17], which, because they are charged, are clear candidates for tetraquark or molecular states.

While the nature of the new structures in charmoinum is not yet understood, the ongoing experimental investigation and theoretical developments make one optimistic that a coherent picture may emerge. The GlueX experiment can build off of this momentum by searching for and study- 


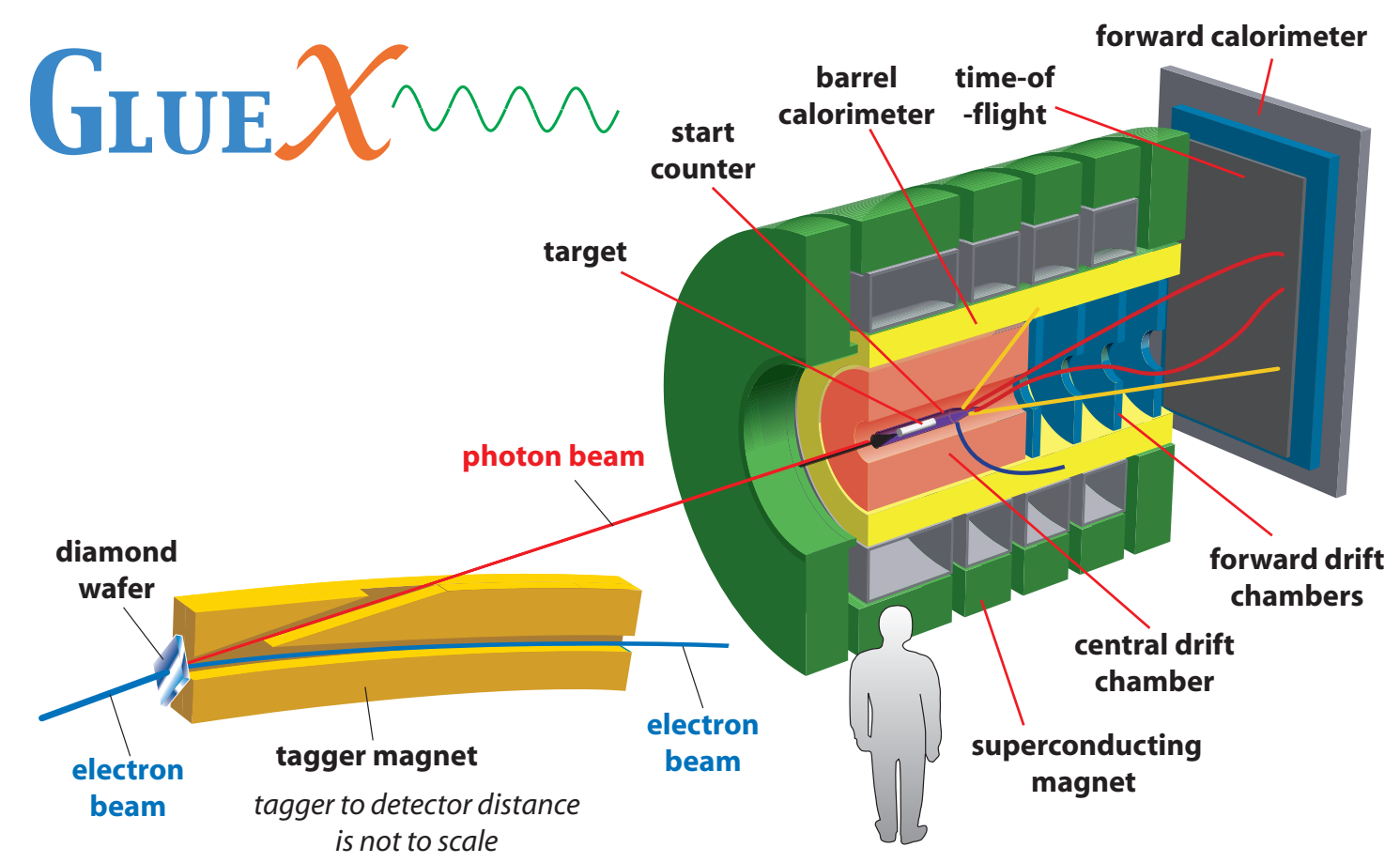

Figure 2: A schematic drawing of the components of the GlueX detector.

ing analogous states in the light quark spectrum. For example, the $Y(2175)[18,19,20]$ has been speculated to be a strangeonium analogue of the $Y(4260)$. For studying hybrids, GlueX has a distinct advantage over $e^{+} e^{-}$experiments: it can produce exotic $J^{P C}$ hybrids directly. (By definition exotics cannot be created directly in a fermion anti-fermion collision.) In addition the use of amplitude analysis and the presence of conventional mesons in the spectrum allows one to construct and interference experiment to study the phase of unknown structures, which is a key input to understanding their nature.

\section{The GlueX Detector}

The GlueX experiment plans to utilize the $12 \mathrm{GeV}$ electron beam available at the newly upgraded Jefferson Lab to produce the $9 \mathrm{GeV}$ high-intensity polarized photon beam necessary to study meson photoproduction. A schematic drawing of the detector appears in Fig. 2. The experimental equipment will be installed in the newly-constructed Hall D at Jefferson Lab.

The photon beam is prepared in a separate tagger building from the experimental hall. The Hall $\mathrm{D}$ tagger receives $12 \mathrm{GeV}$ electron beam that has circulated 5.5 times around the Continuous Electron Beam Accelerator Facility (CEBAF) at Jefferson Lab. Recent upgrades to accelerating modules CEBAF boost the energy per pass to about $2.2 \mathrm{GeV}$, effectively doubling the energy reach of the machine. The $12 \mathrm{GeV}$ beam will be extracted from the machine and will impinge on a $20 \mu \mathrm{m}$ thick diamond radiator. The polarized photon beam is produced via coherent bremsstrahlung radiation. The orientation of the diamond and beam collimation produce a photon beam that has approximately $40 \%$ linear polarization with a peak flux at an energy of about $9 \mathrm{GeV}$. The recoil 
electron is passed through a tagging dipole magnet in order to measure its momentum and consequently, the beam photon energy $E_{\gamma}$. A broadband hodoscope provides $1 \%-2 \%$ resolution on $E_{\gamma}$ from $3.0-11.8 \mathrm{GeV}$, while a movable tagger microscope provides $0.1 \%$ resolution on $E_{\gamma}$ in the coherent radiation peak, which is $8.4-9.0 \mathrm{GeV}$ at $12 \mathrm{GeV}$ primary electron energy.

The linear polarization breaks the azimuthal symmetry of the scattering processing providing an extra observable in the amplitude analysis. In the picture where the reaction proceeds through $t$-channel meson exchange with the target proton, the angular distribution of the production plane is related to the parity of the exchanged particle and the outgoing photoproduced meson. The use of linearly polarized photons in principle therefore provides the capability to study the production mechanism of known mesons or determine the parity of unknown states under assumptions about the production. (For discussion in the context of vector meson production, see Ref. [21].)

Inside of Hall D, the photon beam will collide with a liquid hydrogen target. The the GlueX detector is designed to have a large acceptance for both charged and neutral particles. This should enable exclusive reconstruction of multi-meson final states, which will be critical for performing an amplitude analysis to extract the spin and parity of intermediate hadronic resonances.

The central region of the GlueX detector is immersed in a solenoidal field derived from the superconducting magnet that was utilized previously for the LASS experiment. The planned magnetic field is about 2 Tesla along the beam axis and will enable measurements of the transverse momentum of charged particles. Charged particle tracking is performed by two tracking devices inside the bore of the solenoid. A central drift chamber (CDC) consists of 28 layers of $1.5 \mathrm{~m}$-long straw tubes. The tubes in sixteen of the layers are titled at an angle of $\pm 6^{\circ}$ in order to obtain longitudinal position information. The design resolution of the CDC is $150 \mu \mathrm{m}$ in the $r / \phi$ plane and $2 \mathrm{~mm}$ in $z$ (along the beam axis). The forward drift chamber (FDC) is composed of four six-layer packages. Each layer contains plane anode wires with a plane of cathode strips on either side of the anode wires. The orientation of the strips or wires in cathode or anode is rotated $60^{\circ}$ for each plane with respect to the previous one in order to maximize two-dimensional $(r / \phi)$ position information produced by the layer, i.e., the cathode strips for a particular layer are neither parallel to each other nor to the intermediate anode wires. The design hit resolution in the $r / \phi$ plane is $200 \mu \mathrm{m}$. Combined together the CDC and FDC provide coverage for tracks in a region of polar angle $\theta$ from the beam line of $1^{\circ}<\theta<140^{\circ}$ with a momentum resolution of about $1 \%-3 \%$ over most kinematic regions of interest.

The GlueX detector utilizes a pair of calorimeters to provide reconstruction of photons that are produced in the decays of mesons. A barrel calorimeter (BCAL) with a cylindrical geometry surrounds both the CDC and FDC and sits just inside the inner radius of the magnet bore. The BCAL is constructed from 48 four-meter long modules. It is a sampling calorimeter that utilizes $1 \mathrm{~mm}$ double-clad Kuraray scintillating fibers embedded in a lead and epoxy matrix. The modules are read out on each end by Hamamatsu S12045X MPPC arrays, sometimes called Silicon photomultipliers (SiPM). The SiPMs have an advantage in that they are insensitive to the high magnetic field that exists near due to the proximity of the ends of the BCAL to the flux return of the solenoid magnet. The forward calorimeter is composed of 2800 lead glass blocks that were recycled from the E852 experiment [22]. Electromagnetic showers produce Cherenkov light in the lead glass that is measured with a small FEU 84-3 phototube attached to each block. Together the two calorimeters provide coverage for neutral particles greater than about $50 \mathrm{MeV}$ in the range $2^{\circ}<\theta<120^{\circ}$. 
The approximate energy resolution of both detectors is $6 \% / \sqrt{E}+2 \%$, where $E$ is the photon energy measured in $\mathrm{GeV}$.

Several detectors aid in particle ID and event time determination, which is critical for charged particle tracking and tagging the correct recoil electron that produced the hadronic event of interest. The spacing between bunches of electrons delivered to the diamond radiator by CEBAF is $2 \mathrm{~ns}$. Once a particular bunch is isolated, the event time can be derived very precisely from a global timing signal. Surrounding the target is a $2 \mathrm{~mm}$ thin conically shaped scintillator start counter that delivers about 300 ps - 500 ps resolution for single charged tracks depending on position. In the forward direction, just upstream of the forward calorimeter, is a Time of Flight (TOF) detector composed of two planes of $2.5 \mathrm{~cm}$-thick scintillator that provide $70 \mathrm{ps}$ timing resolution on forwardgoing tracks. Particle identification will be achieved using TOF and specific ionization $(d E / d x)$ in the CDC. The BCAL provides supplemental timing information useful for identifying recoil protons in $\gamma p \rightarrow X p$ collisions. In the present configuration, the GlueX detector is only capable of achieving direct $\pi / K$ discrimination up to momenta of about $2 \mathrm{GeV} / c$. Upgrades to enhance the kaon identification capability are presently under development.

The detector was designed to accommodate a rate of $10^{8}$ beam photons on target per second in the $9 \mathrm{GeV}$ peak of the polarized photon spectrum. This flux corresponds to a hardware (level-1) trigger rate of $200 \mathrm{kHz}$. Of this, about $15 \mathrm{kHz}$ is from inclusive hadronic photoproduction in the $9 \mathrm{GeV}$ region. The entire readout is built with zero-deadtime flash ADCs and multi-hit TDCs to accommodate the large rates. The data bandwidth off the detector at $10^{8} \mathrm{\gamma} / \mathrm{s}$ is anticipated to be $3 \mathrm{~GB} / \mathrm{s}$. A software (level-3) trigger is planned to filter out the hadronic photoproduction events and reduce the final bandwidth to disk by an order of magnitude. It is anticipated that initial running will start 1-2 orders of magnitude below design intensity and gradually increase as rate-capability of the detectors is verified.

As of Spring 2014 all detector systems except the target, start counter, and recoil electron tagger detectors have been assembled and installed in Hall D. The readout electronics is in place and testing of detectors using cosmic rays and builtin monitoring systems has begun. In May 2014 beam was successfully delivered by CEBAF at $10.5 \mathrm{GeV}$ around the machine and into the Hall D tagger beam dump. Detector commissioning with beam is expected to begin in Fall 2014.

\section{GlueX Outlook}

The various phases of the startup of the GlueX physics program, as approved by the Jefferson Lab Physics Advisory Committee, are summarized in Table 1. This phased startup will culminate with full intensity running at $12 \mathrm{GeV}$ in Phase IV. An additional run with enhanced particle identification capability that will emphasize studying hadrons with hidden an open strangeness is being developed. While the schedule is not definite at the present time, tentatively Phase I may commence in 2015 with Phase IV, the full intensity running, starting around 2017-2018.

In order to search for hybrid mesons, one needs to cleanly reconstruct some photoproduction reaction and then perform an amplitude analysis to examine the various resonant contributions. The steps of the process are roughly illustrated in Figs. 3 and 4, which are created from two separate studies using a full hit-level simulation of the GlueX detector and event reconstruction and fitting algorithms. 
Table 1: A table of relevant parameters for the various phases of currently-approved GlueX running (from Ref. [23]. A "PAC day" is 24 hours of continuous beam. Raw data volume projections in Phase IV include the implementation of a level-three trigger.

\begin{tabular}{l|cccc}
\hline \hline & Phase I & Phase II & Phase III & Phase IV \\
\hline Duration (PAC days) & 30 & 30 & 60 & 200 \\
Minimum electron energy $(\mathrm{GeV})$ & 10 & 11 & 12 & 12 \\
Average photon flux $(\gamma / \mathrm{s})$ & $10^{6}$ & $10^{7}$ & $10^{7}$ & $5 \times 10^{7}$ \\
Average beam current $(\mathrm{nA})$ & $50-200$ & 220 & 220 & 1100 \\
Maximum beam emittance $(\mathrm{mm} \cdot \mu \mathrm{r})$ & 50 & 20 & 10 & 10 \\
Level-one (hardware) trigger rate $(\mathrm{kHz})$ & 2 & 20 & 20 & 200 \\
Raw data volume $(\mathrm{TB})$ & 60 & 600 & 1200 & 2300 \\
\hline \hline
\end{tabular}

Suppose one wants to search for the exotic $\pi_{1}(1600) \rightarrow \rho \pi$ reported by COMPASS. One would first attempt to isolate the reaction $\gamma p \rightarrow \pi^{+} \pi^{-} \pi^{+} n$, which corresponds to about $2 \%$ of the total $\gamma p$ hadronic cross section at $E_{\gamma}=9 \mathrm{GeV}$. Figure 3(a) shows the raw $\pi^{+} \pi^{-} \pi^{+}$invariant mass spectrum for all combinations of three pion candidates reconstructed by the detector. One then utilizes a boosted decision tree [25] that is trained to separate signal from background. The BDT in this case is examining the number of tracks, quality of the kinematic fit to the missing neutron mass, the transverse momentum of the missing neutron, the extra energy detected in the calorimeters, and several other variables. When correlations amongst these variables for signal and background are exploited one can develop a classifier that is a strong discriminator of signal and background. The BDT algorithm takes as inputs the quantities noted above and produces a single number that tends toward one for signal and zero for background. This single number provides more signal to background discrimination than any of the individual inputs or by placing separate uncorrelated requirements on each of the inputs. Depending on the desired signal efficiency and purity, a minimum requirement is placed on the value of this classifier. Requiring $95 \%$ purity produces the distribution in Fig. 3(b).

Once a clean sample has been isolated, an amplitude analysis can be performed to attempt to decompose the $\pi^{+} \pi^{-} \pi^{+}$system into various resonant components. In this study, the $3 \pi$ spectrum was assumed to be populated by five different resonant decays: $a_{1} \rightarrow \rho \pi, a_{2} \rightarrow \rho \pi, \pi_{2} \rightarrow f_{2} \pi$, $\pi_{2} \rightarrow \rho \pi$, and the exotic $\pi_{1} \rightarrow \rho \pi$, all of which result in production of three pions. As a test of sensitivity of GlueX detector, the exotic amplitude was generated at about $2 \%$ of the entire $3 \pi$ mass distribution (a level consistent with the upper limit reported by CLAS at $6 \mathrm{GeV}$ [12]). Of course, one hopes to find decay channels, like $\eta^{\prime} \pi$ in pion production, where the exotic amplitude is at the same scale as conventional decays. By fitting the various angular distributions of the decay, one can determine the magnitude and phase of each of these components in a single bin of $3 \pi$ invariant mass. Such a fit is repeated for all bins of $3 \pi$ mass, each of which is statistically independent of the others. (Binning the data in $3 \pi$ invariant mass makes one insensitive to modeling the lineshape of the produced resonance in the analysis, which is ideal if one is searching for states with unknown properties.) The top panel of Fig. 4 shows the decomposition of the spectrum into the various resonant components. The colored markers show the fit results for the $1^{++}, 2^{++}, 1^{-+}$, and two 

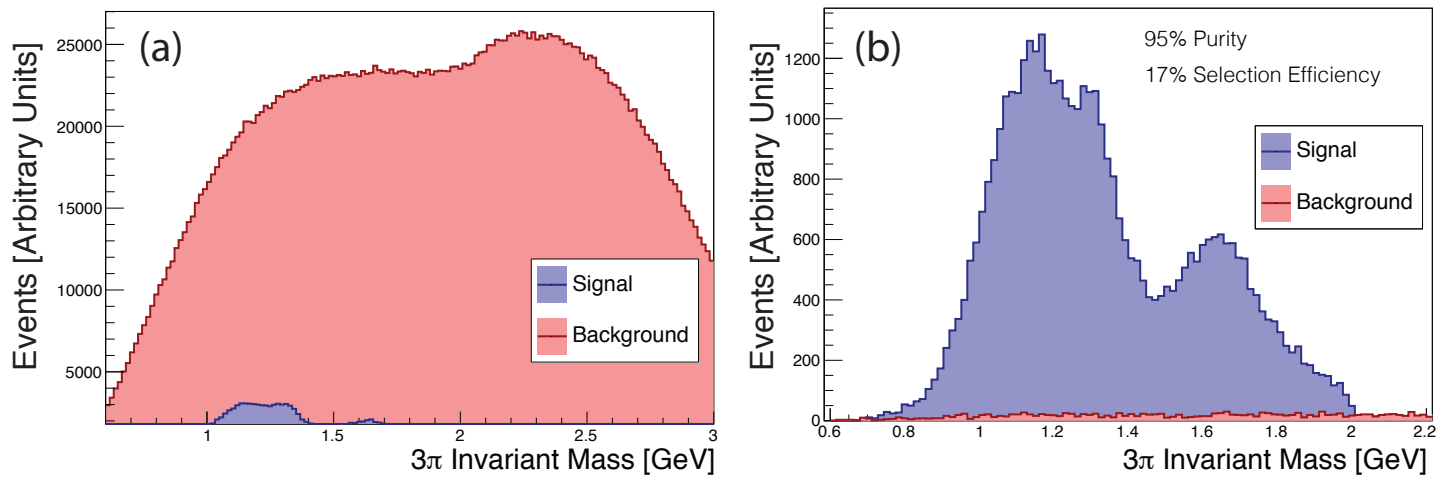

Figure 3: (a) A Monte Carlo simulation of the reconstructed spectrum of $\pi^{+} \pi^{+} \pi^{-}$invariant mass for all combinations of three pions produced and detected in inclusive $\gamma p$ collisions. No specific event selection criteria have been defined. (b) The same spectrum as in (a) after requiring that the boosted-decision tree classifier trained to select $\gamma p \rightarrow \pi^{+} \pi^{-} \pi^{+} n$ exceed some minimum value that generates a $95 \%$ pure sample; $17 \%$ of the signal in (a) meet this criteria [24].

$2^{-+}$amplitudes, which, in this simple study, are populated each only by one resonance: $a_{1}(1230)$, $a_{2}(1320), \pi_{1}(1600)$, and $\pi_{2}(1670)$. The solid black line shows the sum of all amplitudes and is equal to the efficiency-corrected $3 \pi$ invariant mass distribution.

The bottom panel shows the sensitivity to the small exotic amplitude. One also sees a feature of amplitude analysis: the unique phase signature of a resonance can be extracted by measuring the interference of amplitudes, in this case the $\pi_{1}$ and $a_{1}$ decays.

The example above illustrates the capability of the GlueX detector to both reconstruct and perform an amplitude analysis of the $\pi^{+} \pi^{-} \pi^{+}$system, in a single photoproduction reaction. A large number of other final states including almost any combination of several neutral and charged mesons are accessible for study. In addition, the large acceptance multi-purpose GlueX detector makes it broadly useful for a variety of physics topics beyond hybrid meson spectroscopy. There are existing plans to study Primakoff production of light pseudoscalars and searches for $\Xi$ baryons. Proposals that require augmenting detector capability are being developed to study rare decays of $\eta$ mesons and pion polarizability. Given that so little high statistics photoproduction data exist, it is expected that even the first phases of GlueX running will open a door to a wide variety of new physics topics.

To set the scale for the expected event yields in GlueX, a useful rule of thumb is that an intensity of $10^{7} \mathrm{\gamma} / \mathrm{s}$ will produce about $10^{6}$ events per $1 \mu \mathrm{b}$ of cross section per day of running. The total hadronic cross section at $9 \mathrm{GeV}$ is about $120 \mu \mathrm{b}$. Therefore at design intensity of $10^{8} \gamma / \mathrm{s}$, 100 days of running will produce $10^{11}$ hadronic events. While many cross sections are unknown, most reactions of interest likely have cross sections at the $0.1 \mu \mathrm{b}-1 \mu \mathrm{b}$ level. Therefore, even at the initial phases and after detection and selection efficiencies, one expects of order $10^{5}-10^{6}$ analyzable events in many reactions. 


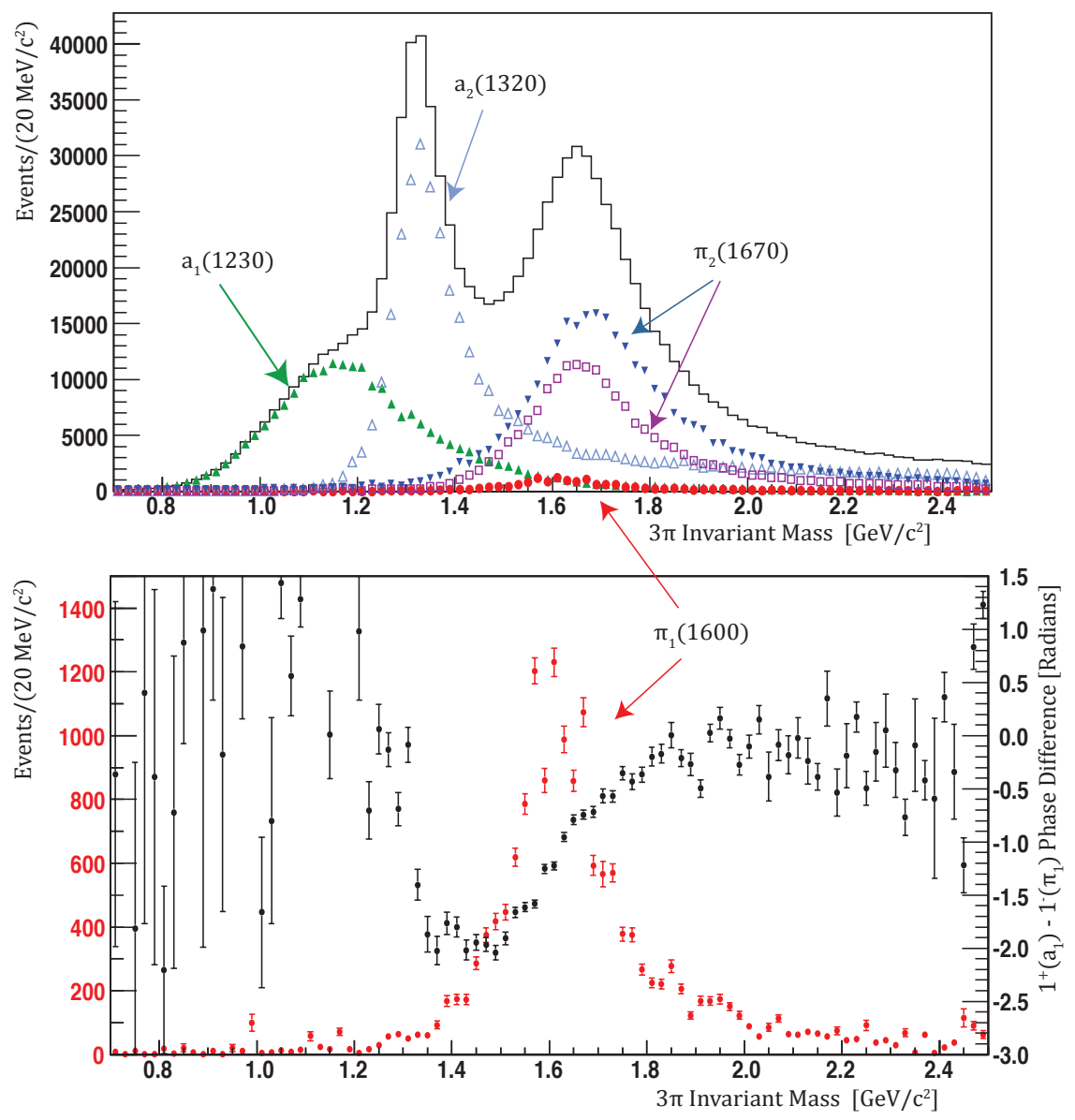

Figure 4: An amplitude analysis of simulated data in which various resonant decays populate the $3 \pi$ mass spectrum (shown by the solid line in the top plot). The top plot shows the magnitudes of the various components in each bin as extracted by an amplitude analysis. The bottom plot shows a zoom of the exotic amplitude and the phase of the exotic with respect to the $a_{1} \rightarrow \rho \pi$ decay.

\section{Summary}

The GlueX experiment is plans to utilize the unique features of the $12 \mathrm{GeV}$ upgrade at Jefferson Lab to conduct a search for hybrid mesons using polarized photoproduction, a production mechanism that is relatively unexplored in this energy regime and complementary to existing hadron beam data. The construction of the detector itself is nearly complete; first commissioning with beam is expected in the fall of 2014. The large acceptance of the detector allows one at access a variety of different and complementary meson decay modes. In the next few years, even in the early phases of GlueX running, one will have the opportunity to study meson photoproduction and search for hybrid mesons with unprecedented statistical precision. 


\section{Acknowledgements}

The author would like to thank the organizers of the Bormio winter meeting on nuclear physics for both the invitation to attend and present this work and for organizing a productive and enjoyable meeting. The author would also like to acknowledge the useful comments and discussion from fellow collaborators on the GlueX experiment. This work was supported by the U.S. Department of Energy Office of Nuclear Physics under grant DE-FG02-05ER41374.

\section{References}

[1] E. Klempt and A. Zaitsev, Phys. Rept. 454, 1 (2007) [arXiv:0708.4016 [hep-ph]].

[2] R. L. Jaffe and K. Johnson, Phys. Lett. B 60, 201 (1976).

[3] T. Barnes, F. E. Close, F. de Viron and J. Weyers, Nucl. Phys. B 224, 241 (1983).

[4] M. S. Chanowitz and S. R. Sharpe, Nucl. Phys. B 222, 211 (1983) [Erratum-ibid. B 228, 588 (1983)].

[5] N. Isgur and J. E. Paton, Phys. Rev. D 31, 2910 (1985).

[6] J. J. Dudek, Phys. Rev. D 84, 074023 (2011) [arXiv:1106.5515 [hep-ph]].

[7] F. E. Close and J. J. Dudek, Phys. Rev. Lett. 91, 142001 (2003) [hep-ph/0304243].

[8] C. A. Meyer and Y. Van Haarlem, Phys. Rev. C 82, 025208 (2010) [arXiv:1004.5516 [nucl-ex]].

[9] E. I. Ivanov et al. [E852 Collaboration], Phys. Rev. Lett. 86, 3977 (2001) [hep-ex/0101058].

[10] G. S. Adams et al. [CLEO Collaboration], Phys. Rev. D 84, 112009 (2011) [arXiv:1109.5843 [hep-ex]].

[11] M. Alekseev et al. [COMPASS Collaboration], Phys. Rev. Lett. 104, 241803 (2010) [arXiv:0910.5842 [hep-ex]].

[12] M. Nozar et al. [CLAS Collaboration], Phys. Rev. Lett. 102, 102002 (2009) [arXiv:0805.4438 [hep-ex]].

[13] G. T. Bodwin, E. Braaten, E. Eichten, S. L. Olsen, T. K. Pedlar and J. Russ, arXiv:1307.7425.

[14] R. E. Mitchell, PoS Hadron 2013, 038 (2014).

[15] L. Liu et al. [Hadron Spectrum Collaboration], JHEP 1207, 126 (2012) [arXiv:1204.5425 [hep-ph]].

[16] M. Ablikim et al. [BESIII Collaboration], Phys. Rev. Lett. 110, no. 25, 252001 (2013) [arXiv:1303.5949 [hep-ex]].

[17] M. Ablikim et al. [BESIII Collaboration], Phys. Rev. Lett. 111, 242001 (2013) [arXiv:1309.1896 [hep-ex]].

[18] B. Aubert et al. [BABAR Collaboration], Phys. Rev. D 74, 091103 (2006) [hep-ex/0610018].

[19] M. Ablikim et al. [BES Collaboration], Phys. Rev. Lett. 100, 102003 (2008) [arXiv:0712.1143 [hep-ex]].

[20] C. P. Shen et al. [Belle Collaboration], Phys. Rev. D 80, 031101 (2009) [arXiv:0808.0006 [hep-ex]].

[21] K. Schilling, P. Seyboth and G. E. Wolf, Nucl. Phys. B 15, 397 (1970) [Erratum-ibid. B 18, 332 (1970)].

[22] R. R. Crittenden et al., Nucl. Instrum. Meth. A 387, 377 (1997). 
[23] A. AlekSejevs et al. [GlueX Collaboration], arXiv:1305.1523 [nucl-ex].

[24] T. Boettcher, "Applying machine learning to GlueX data analysis," Presentation at the American Physical Society Meeting, Savannah, GA (2014).

[25] A. Hoecker, et. al, "TMVA: Toolkit for Multivariate Data Analysis,Ó PoS A CAT 040 (2007). 